\title{
Algebraic IDA-PBC for Polynomial Systems with Input Saturation: An SOS-based Approach
}

\author{
Cieza A., Oscar B. ${ }^{1}$; Reger, Johann ${ }^{2}$ \\ ${ }^{1}$ Technische Universität Ilmenau, Grupo de Ingeniería de Control, Ilmenau, Alemania
}

\begin{abstract}
The necessity to deal with partial differential equations (PDEs) and the dissipation condition are the main adversities in the application of Interconnection and Damping Assignment Passivity-Based Control (IDA-PBC). Recently, an algebraic solution of IDA-PBC has been explored for a class of affine polynomial systems by using sum of squares (SOS) and semidefinite programming (SDP). In this work, we extend the previous method by incorporating actuator saturation (AS) and two minimization objectives in the SDP. Our results are validated on two polynomial systems.
\end{abstract}

Keywords: Port-Hamiltonian Systems, IDA-PBC, Polynomial Systems, Sum of Squares, Actuator Saturation.

\section{IDA-PBC Algebraico Para Sistemas Polinomiales con Saturación en las Entradas: Un Enfoque Basado en SOS}

\begin{abstract}
Resumen: La solución de ecuaciones diferenciales parciales (PDE) y la condición de disipación son las principales adversidades en la aplicación de Interconnection and Damping Assignment Passivity-Based Control (IDA-PBC). Recientemente, se ha explorado una solución algebraica de IDA-PBC para una clase de sistemas polinomiales utilizando el método de suma de cuadrados (SOS) y la programación semidefinida (SDP). En este trabajo se amplía el método anterior incorporando saturación en los actuadores (AS) y dos objetivos de minimización en la SDP. Nuestros resultados son validados en dos sistemas polinómicos.
\end{abstract}

Palabras claves: Sistemas Port-Hamiltonianos, IDA-PBC, Sistemas Polinomiales, Suma de Cuadrados, Saturación del Actuador.

\section{INTRODUCTION}

Over the last decade, Interconnection and Damping Assignment Passivity-Based Control (IDA-PBC) has experienced increasing practice due its wide applicability (Petrović et al., 2001; Batlle et al., 2004, 2007; Ortega and García-Canseco, 2004; Li et al., 2010; Astolfi and Ortega, 2001; Fujimoto et al., 2001; Renton et al., 2012; Li et al., 2013; Astolfi et al., 2002a; Xue and Zhiyong, 2017). The standard IDA-PBC method requires a two step procedure: energy shaping and damping injection. The first depends on the solution of partial differential equations (PDEs) and the second together with zero state detectability (ZSD) of the closed-loop guarantees asymptotic stability.

In order to simplify the PDE problem Viola et al. (2007) have introduced a change of coordinates and a modification of the target dynamics. With the objective to completely avoid PDEs, the following leading methods have been proposed: constructive procedures (Donaire et al., 2016a; Borja et al., 2016; Romero et al., 2017), implicit port-Hamiltonian representation (Macchelli, 2014; Castaños and Gromov, 2016) and an algebraic approach (Fujimoto and Sugie, 2001; Batlle et al., 2007; Nunna et al., 2015). In addition, it has been shown in (Batlle et al., 2007;
Donaire et al., 2016b) that a two step IDA-PBC may be restrictive in some cases, thus introducing a single step procedure (SIDAPBC). Furthermore, dissipation in the under-actuated degrees of freedom, see (Gómez-Estern and Van der Schaft, 2004), may also turn out an obstacle for the implementation of IDA-PBC on some systems, e.g. on the cart-pole system (Delgado and Kotyczka, 2014).

It is well-known that actuator saturation (AS) can cause performance losses or even lead to closed-loop instability. In this context, Åström et al. (2008); Escobar et al. (1999) have studied PBC with AS on two specific systems. Sun et al. (2009); Wei and Yuzhen (2010) analyze stability for saturation in the damping injection term. A variable structure approach to energy shaping for a class of Port-Hamiltonians system is developed in (Macchelli, 2002; Macchelli et al., 2003). Besides, Sprangers et al. (2015) studied a reinforcement learning method for energy shaping which shows robust properties under AS.

In polynomial systems the sum of squares (SOS) approach with semidefinite programming (SDP) allows to synthesize Lyapunov functions (Parrilo, 2000), optimal (Ichihara, 2009), robust (Zhu et al., 2018; Jennawasin et al., 2010), fuzzy (Wibowo et al., 2014; 
Yu and Wang, 2013) and AS-focused controllers (Jennawasin et al., 2012; Valmorbida et al., 2013; Ichihara, 2013), among others. This AS controllers, calculated with SOS, use the polytope representation introduced in ( $\mathrm{Hu}$ and $\mathrm{Lin}, 2001)$ for linear system with multiple input saturation.

For a class of polynomial affine systems, lately, Cieza and Reger (2018) have presented an algebraic method the conditions of which are met by means of SOS and SDP solutions. The method solves the typical problems of IDA-PBC at the expense of an adequate parametrization and selection of the Hamiltonian. To the best knowledge of the authors there is no definitive solution to the AS controller design problem with IDA-PBC. The underlying work shall now also incorporate AS and two minimization objectives in the SDP solver, extending the algorithm of Cieza and Reger (2018).

The work is organized as follows. We summarize the concepts of IDA-PBC for nonlinear affine systems in Section 2. Section 3 recapitulates the algebraic method of Cieza and Reger (2018). In Section 4 we solve the AS problem in the algebraic approach using additionally two minimization objectives. We discuss the application of SOS methodology and verify our results in Section 5, applying the approach on two polynomial systems. Finally, we draw our conclusions in Section 6.

\section{IDA-PBC FOR AFFine Nonlinear Systems}

Let us recall the IDA-PBC approach for nonlinear affine systems introduced by Ortega and García-Canseco (2004). Consider the system

$$
\dot{x}=f(x)+g(x) u
$$

and the target port-Hamiltonian system

$$
\dot{x}=F_{d}(x)\left(\frac{\partial H_{d}}{\partial x}\right)^{\top}(x),
$$

where $\mathscr{X}_{h} \subset \mathbb{R}^{n_{x}}$ is the state space manifold, $\mathscr{U} \subset \mathbb{R}^{m}$ is the input space, $g$ has full rank, the skew symmetric portion $\frac{1}{2}\left(F_{d}-F_{d}^{\top}\right) \in \mathbb{R}^{n_{x} \times n_{x}}$ is the interconnection matrix, the symmetric portion $\mathbb{R}^{n_{x} \times n_{x}} \ni \frac{1}{2}\left(F_{d}+F_{d}^{\top}\right) \preceq 0$ is the dissipation matrix and $H_{d}: \mathscr{X}_{h} \rightarrow \mathbb{R}$ with $x^{\star}=\operatorname{argmin} H_{d}(x)$ is the desired positive definite Hamiltonian. If the matching condition ${ }^{1}$

$$
g_{\perp}(x) f(x)=g_{\perp}(x) F_{d}(x)\left(\frac{\partial H_{d}}{\partial x}\right)^{\top}(x)
$$

is fulfilled for some $F_{d}, H_{d}$ and full rank left annihilator ${ }^{2} g_{\perp}$ then the control law

$$
u_{I}=\left(g^{\top}(x) g(x)\right)^{-1} g^{\top}(x)\left(F_{d}(x)\left(\frac{\partial H_{d}}{\partial x}\right)^{\top}(x)-f(x)\right)
$$

transforms (1) into the stable system (2) with Lyapunov function $H_{d}$. Asymptotic stability of $x^{\star}$ in the attainable set $\mathscr{X}_{a}=\left\{x \in \mathscr{X}_{h} \mid\right.$

\footnotetext{
${ }^{1}$ If in the matching condition (3) the structure of $F_{d}(x)$ is fixed, then (3) is a PDE. If $H(x)$ is fixed, then (3) is an algebraic equation.

${ }^{2}$ The full rank left annihilator $g_{\perp}$ is given by $g_{\perp}(x) g(x)=0$ and $\operatorname{rank}\left(g_{\perp}\right)=$ $n_{x}-m$. Consequently, (3) exists iff $n_{x}>m$.
}

$\left.g_{\perp} f=0\right\}$ may be demonstrated e.g. by using passivity with Lyapunov stability theory, see (Astolfi et al., 2002b; Sepulchre et al., 1997).

\section{IDA-PBC For Polynomial Systems}

In this section we summarize Proposition 4-5 from (Cieza and Reger, 2018) for $\beta=1$. The variable $\beta$ of the aforementioned work can be considered as a scaling factor, which does not alter our main results.

\subsection{Algebraic IDA-PBC}

Let $\Gamma(x), g(x), g_{\perp}(x)$, and $g_{\perp}(x) f(x)$ be polynomial functions in

$$
\Gamma(x) \dot{x}=f(x)+g(x) u
$$

and the desired closed loop port-Hamiltonian system

$$
\Gamma(x) \dot{x}=\left[\begin{array}{l}
g_{\perp}(x) \\
g^{\top}(x)
\end{array}\right]^{-1} F(x)\left(\frac{\partial H_{d}}{\partial x}\right)^{\top}(x),
$$

where

$$
F(x)=\left[\begin{array}{l}
F_{1}(x) \\
F_{2}(x)
\end{array}\right], \quad H_{d}(x)=z^{\top}(x) P^{-1} z(x), \quad P=P^{\top} \succ 0 .
$$

Without loss of generality let $\operatorname{argmin} H_{d}(x)=0$. Besides, state and input spaces remain as in (1), z: $\mathscr{X}_{h} \rightarrow \mathbb{R}^{n_{z}}$ is a vector of polynomials with $n_{z} \geq n_{x}, P \in \mathbb{R}^{n_{z} \times n_{z}}$ is a constant matrix, $F, \Gamma$ : $\mathscr{X}_{h} \rightarrow \mathbb{R}^{n_{x} \times n_{x}}$ are full rank polynomial matrices ${ }^{3}$, and $F$ holds the portions $F_{2}(x) \in \mathbb{R}^{m \times n_{x}}$ and $F_{1}(x) \in \mathbb{R}^{n_{x}-m \times n_{x}}$.

Proposition 1 Closing the loop of system (4) with control

$$
u_{b}=\left(g^{\top} g\right)^{-1}\left(F_{2}\left(\frac{\partial z}{\partial x}\right)^{\top} P^{-1} z-g^{\top} f\right)
$$

renders the equilibrium point $x^{\star}=0$ of the closed loop system (5) locally stable for any initial state $x(0)=x_{0} \in \mathscr{X}_{P}=\{x \in$ $\left.\mathbb{R}^{n_{x}} \mid H_{d}(x)=z^{\top}(x) P^{-1} z(x) \leq 1\right\}$ whenever the following conditions are fulfilled for all $x \in \mathscr{X}_{h}=\left\{x \in \mathbb{R}^{n_{x}} \mid 0 \leq h(x)=\right.$ $\left.1-z^{\top}(x) S_{h}^{-1} z(x), S_{h} \succ 0\right\}:$

C1 There exist polynomial functions $\Lambda_{1}(x), g_{\perp}(x)$ and $z(x)$ such that $g_{\perp}(x) f(x)=\Lambda_{1}(x) z(x)$ and $z(x)=0$ iff $x=0$. Besides, if $n_{z}=n_{x}$ then $\frac{\partial z}{\partial x}$ is unimodular ${ }^{4}$, else $\frac{\partial z}{\partial x}$ has rank $n_{x}$ and $\left(\frac{\partial z}{\partial x}\right)_{\perp}(x) P \Lambda_{1}^{\top}(x)=0$ with $\left(\frac{\partial z}{\partial x}\right)_{\perp}$ the full rank left annihilator of the Jacobian of $z$.

C2 There is a polynomial function $N(x)$ such that if $n_{z}=n_{x}$ then $N(x)=\left(\frac{\partial z}{\partial x}\right)^{-\top}(x)$, else $\left(\frac{\partial z}{\partial x}\right)^{\top}(x) N(x)=I_{n_{x}}$ and $\left[N\left(\frac{\partial z}{\partial x}\right)_{\perp}^{\top}\right]$ is a full rank square matrix. ${ }^{5}$

\footnotetext{
${ }^{3}$ Invertibility of $\Gamma$ enables (4) to take the usual form $\dot{x}=\bar{f}(x)+\bar{g}(x) u$ and $F_{d}(x)=\Gamma^{-1}(x)\left[\begin{array}{l}g_{\perp}(x) \\ g^{\top}(x)\end{array}\right]^{-1} F(x)$.

${ }^{4}$ Polynomial matrices are unimodular if the inverse matrix again is a polynomial matrix. Their determinant always is a non-zero constant.

${ }^{5}$ Here $I_{n}$ represents the identity matrix of size $n$.
} 
C3 There is a constant matrix $P$, and polynomial matrices $0 \preceq$ $S_{1}(x) \in \mathbb{R}^{n_{x} \times n_{x}}$ and $F_{2}(x)$ such that

$$
\begin{gathered}
-\Phi(x)-\Phi^{\top}(x)-h(x) S_{1}(x) \succeq 0, \\
S_{h} \succeq P \succeq z\left(x_{0}\right) z^{\top}\left(x_{0}\right), \\
\Phi=\left[\begin{array}{l}
g_{\perp} \\
g^{\top}
\end{array}\right] \Gamma\left[\begin{array}{ll}
F_{1}^{\top} & \left.F_{2}^{\top}\right],
\end{array} F_{1}(x)=\Lambda_{1}(x) P N(x) .\right.
\end{gathered}
$$

Furthermore, the origin of (5) is asymptotically stable if

$$
-\Phi(x)-\Phi^{\top}(x)-h(x) S_{1}(x) \succ 0 \quad \text { and }
$$

for $n_{z}>n_{x},\left(\frac{\partial z}{\partial x}\right)^{\top}(x) P^{-1} z(x)=0$ implies $x=0$.

Proof 1 It can be found in (Cieza and Reger, 2018) with a modification of (8) using the Schur complement.

\subsection{Existence of $u_{b}$}

The next proposition provides a sufficient condition for the existence of $F_{2}$, i.e. the existence of the (asymptotically) stabilizing control law (6).

Proposition 2 Consider $x_{0} \in \mathscr{X}_{P} \subset \mathscr{X}_{h}$ and assume $\Lambda_{1}, g_{\perp}, z, N$ are selected according to $C 1$ and $C 2$. Then there exists a function $F_{2}(x)$ that meets $C 3$ if there exists $P=P^{\top} \succ 0$ and $S_{2}(x) \succeq 0$ such that

$$
\begin{gathered}
-\phi(x)-\phi^{\top}(x)-h(x) S_{2}(x) \succeq 0, \\
\phi(x)=g_{\perp}(x) \Gamma(x) F_{1}^{\top}(x)=g_{\perp}(x) \Gamma(x) N^{\top}(x) P \Lambda_{1}^{\top}(x),
\end{gathered}
$$

with $\phi(x), S_{2}(x) \in \mathbb{R}^{n_{x}-m \times n_{x}-m}$. Additionally, (9) is solvable as long as

$$
-\phi(x)-\phi^{\top}(x)-h(x) S_{2}(x) \succ 0 .
$$

Lastly, if (10) or (11) are satisfied, then a solution for $F_{2}$ with $0 \succ L(x)+L^{\top}(x) \in \mathbb{R}^{m \times m}$ is

$$
F_{2}=\left[-g^{\top}(x) \Gamma(x) F_{1}^{\top}(x), \quad L(x)\right]\left[\begin{array}{l}
g_{\perp}(x) \\
g^{\top}(x)
\end{array}\right]^{-\top} \Gamma^{-\top}(x)
$$

Proof 2 See (Cieza and Reger, 2018, Prop. 5).

Application of the algorithm starts with adequate selection of $\Lambda_{1}$, $z, g_{\perp}$ and $N$ according to (4), (5), C1 and C2. Later, we choose $h$ and solve (for convenience) (7), (9), (10) or (11) searching for $P$ (and $F_{2}$ in case of Prop. 1) under (8) which defines an upper and lower bound on $P$, namely $x_{0} \in \mathscr{X}_{P} \subset \mathscr{X}_{h}$ for some given $x_{0}$.

In comparison, Proposition 2 requires the solution of smaller LMIs to calculate the parametrized function $F_{2}$, see (12), or to guarantee its existence, whereas Prop. 1 defines $F_{2}$ as general function s.t. (7) (and (9)) is satisfied, which grants more flexibility at the expense of computational cost. In order to use SOS with SDP we force $F_{2}$ (in Prop. 1) to be a polynomial function of some selected degree.

Proposition 2 can also be used as a fast indicator such that Proposition 1 will work. Note that Proposition 2 contains the minimal conditions that $P, \Lambda_{1}, g_{\perp}, N, h$ and $\Gamma$ have to satisfy, and it guarantees the existence of a not necessarily polynomial function $F_{2}$. Hence, if we constrain $F_{2}$ to be polynomial, then Proposition 2 is experimentally still a good, but not an unconditionally reliable reference.

\section{Main Result}

In view of Proposition 1 and 2, we extend the results of Cieza and Reger (2018) to consider actuator saturation (AS). In addition, we define two possible minimizations (optimization objectives) for the SDP.

\subsection{Actuator Saturation (AS)}

Proposition 3 Let all conditions of Prop. 1 for local (asymptotic) stability be satisfied and assume:

C5 There exist polynomial matrices $\Lambda_{2}(x) \in \mathbb{R}^{m \times n_{z}}$ and $0 \preceq$ $S_{3}(x) \in \mathbb{R}^{m \times m}$ such that

$$
\begin{gathered}
{\left[\begin{array}{c}
\Lambda_{1}(x) \\
\Lambda_{2}(x)
\end{array}\right] z(x)=\left[\begin{array}{l}
g_{\perp}(x) \\
g^{\top}(x)
\end{array}\right] f(x),} \\
{\left[\begin{array}{ll}
\eta_{11}(x) & \eta_{12}(x) \\
\eta_{12}^{\top}(x) & P
\end{array}\right] \succeq 0,} \\
\eta_{11}(x)=\left(g^{\top} g\right)(x) S_{u}(x)\left(g^{\top} g\right)(x)-h(x) S_{3}(x), \\
\eta_{12}(x)=F_{2}(x)\left(\frac{\partial z}{\partial x}\right)^{\top}(x)-\Lambda_{2}(x) P .
\end{gathered}
$$

Then the stabilizing control law (6) is restricted to $\mathscr{U}_{b}=\left\{u_{b} \in\right.$ $\left.\mathscr{U} \mid u_{b}^{\top} S_{u}^{-1}(x) u_{b} \leq 1\right\}$ with $\mathbb{R}^{m \times m} \ni S_{u}(x)=S_{u}^{\top}(x) \succ 0$ for any $x \in \mathscr{X}_{P}$.

Proof 3 Multiplying (14) on both sides by adequate matrices and using the Schur complement yields

$$
I_{m}-W W^{\top} \succeq h S_{u}^{-\frac{1}{2}}\left(g^{\top} g\right)^{-1} S_{3}\left(g^{\top} g\right)^{-1} S_{u}^{-\frac{1}{2}}
$$

with $W(x)=S_{u}^{-\frac{1}{2}}\left(g^{\top} g\right)^{-1}\left(F_{2}\left(\frac{\partial z}{\partial x}\right)^{\top} P^{-\frac{1}{2}}-\Lambda_{2} P^{\frac{1}{2}}\right)$ and $P^{\frac{1}{2}} P^{\frac{1}{2}}=$ $P$. Now taking the spectral norm on (15) for $x \in \mathscr{X}_{P} \subset \mathscr{X}_{h}$, i.e. $h \geq 0$, and the definition of $\mathscr{X}_{P}$ as $\left\|P^{-\frac{1}{2}} z\right\|_{2}^{2} \leq 1$ we have

$$
\begin{aligned}
1 & \geq\left\|S_{u}^{-\frac{1}{2}}\left(g^{\top} g\right)^{-1}\left(F_{2}\left(\frac{\partial z}{\partial x}\right)^{\top} P^{-\frac{1}{2}}-\Lambda_{2} P^{\frac{1}{2}}\right)\right\|_{2}^{2} \\
& \geq\left\|S_{u}^{-\frac{1}{2}}\left(g^{\top} g\right)^{-1}\left(F_{2}\left(\frac{\partial z}{\partial x}\right)^{\top} P^{-\frac{1}{2}}-\Lambda_{2} P^{\frac{1}{2}}\right)\right\|_{2}^{2}\left\|P^{-\frac{1}{2}} z\right\|_{2}^{2} \\
& \geq\left\|S_{u}^{-\frac{1}{2}}\left(g^{\top} g\right)^{-1}\left(F_{2}\left(\frac{\partial z}{\partial x}\right)^{\top} P^{-1} z-\Lambda_{2} z\right)\right\|_{2}^{2} \\
& =\left\|S_{u}^{-\frac{1}{2}} u_{b}\right\|_{2}^{2}=u_{b}^{\top} S_{u}^{-1} u_{b},
\end{aligned}
$$

where last equalities are obtained with (13) and (6).

After solving the conditions of Proposition 1 and 3, we may calculate a control input $u_{b} \in \mathscr{U}_{b}$, for any $x \in \mathscr{X}_{P}$. Proposition 3 can 
also be extended to work with Prop. 2 by replacing (12) in (14). This yields an LMI which is not necessarily polynomial. Therefore, we restrict $L$ to be polynomial and multiply (14) on the right with the square non-singular matrix ${ }^{6}$

$$
\left[\begin{array}{cccc}
I_{m} & 0 & 0 & 0 \\
0 & N(x) \Gamma^{\top}(x) g_{\perp}^{\top}(x) & N(x) \Gamma^{\top}(x) g(x) & \left(\frac{\partial z}{\partial x}\right)_{\perp}^{\top}(x)
\end{array}\right]
$$

and on the left by its transpose. This results in conditions that can be solved by means of SOS + SDP.

Following the works of $\mathrm{Hu}$ and Lin (2001); Valmorbida et al. (2013); Ichihara (2013), among others, we may use the polytope or polytopic saturation model within the algebraic IDA-PBC, as phrased in the following proposition.

Proposition 4 Let the conditions of Propositions 1 and 3 be satisfied for some system of the form (4) resulting in some matrices $P, F_{2}$ and a locally (asymptotically) stabilizing constrained controller $u=u_{b} \in \mathscr{U}_{b}$ given by (6). Consequently, there is a new (asymptotically) stabilizing control action

$$
\begin{array}{r}
u_{s} \in \mathscr{U}_{s}=\left\{u_{s}=u_{b}+\left(g^{\top} g\right)^{-1} \Theta u_{\delta} \mid \theta_{k} \in[0,1]\right\}, \\
u_{\delta}=\left(F_{21}-F_{20}\right)\left(\frac{\partial z}{\partial x}\right)^{\top} P^{-1} z, \quad F_{2}=F_{20},
\end{array}
$$

provided that there exist matrices $F_{21}(x) \in \mathbb{R}^{m \times n_{z}}$ and $\bar{S}_{i_{1} \ldots i_{m}}(x) \succeq$ 0 , s.t. for all $i_{k} \in\{0,1\}$ with $k=1 \ldots m$,

$$
\begin{gathered}
-\Phi_{i_{1} \ldots i_{m}}(x)-\Phi_{i_{1} \ldots i_{m}}^{\top}(x)-h(x) \bar{S}_{i_{1} \ldots i_{m}}(x) \succeq 0, \\
\Phi_{i_{1} \ldots i_{m}}=\left[\begin{array}{l}
g_{\perp} \\
g^{\top}
\end{array}\right] \Gamma\left[\begin{array}{llll}
F_{1}^{\top} & F_{2 i_{1}}^{\top}(x) e_{1} & \ldots & F_{2 i_{m}}^{\top}(x) e_{m}
\end{array}\right],
\end{gathered}
$$

where $\Theta=\operatorname{diag}\left(\theta_{1}, \ldots, \theta_{m}\right)$ and $e_{i}$ the ith unity vector. In addition, asymptotic stability is achieved if (9) are satisfied and (17) is strict.

\section{Proof 4 Define}

$$
F_{2}=\Theta F_{21}+\left(I_{m}-\Theta\right) F_{20}, \quad \text { (polytope) }
$$

and $\beta_{k 0}+\beta_{k 1}=1, \theta_{k}=\beta_{k 0}$, then (16) follows from (6) and (18). Then, replace (18) in (7), multiply it by $\sum_{i_{k}=0}^{1} \beta_{k i_{k}}$ for convenience and substitute $S_{1}$ with $\bar{S}_{i_{1} \ldots i_{m}}$ which does not affect stability, see (Cieza and Reger, 2018). Then (7) results in

$$
0 \preceq \sum_{i_{1}=0}^{1} \beta_{1 i_{1}} \ldots \sum_{i_{m}=0}^{1} \beta_{m i_{m}}\left(-\Phi_{i_{1} \ldots i_{m}}-\Phi_{i_{1} \ldots i_{m}}^{\top}-h \bar{S}_{i_{1} \ldots i_{m}}\right),
$$

and rewritten as a sum of positive semidefinite polynomial functions (convex set) this yields

$$
0 \preceq \sum_{j=0}^{2^{m}-1} \bar{\beta}_{j}\left(-\Phi_{j}-\Phi_{j}^{\top}-h \bar{S}_{j}\right),
$$

with $j=\sum_{k=1}^{m} i_{k} 2^{k-1}, \sum_{j=0}^{2^{m}-1} \bar{\beta}_{j}=1$. Therefore, a sufficient condition for (19) is (17). The proof of asymptotic stability follows a similar procedure.

\footnotetext{
${ }^{6}$ Note that using $\mathrm{C} 2$, the square matrix $\left[N\left(\frac{\partial z}{\partial x}\right)_{\perp}^{\top}\right]$ and as a consequence $\left[N \Gamma^{\top} g_{\perp}^{\top}, N \Gamma^{\top} g,\left(\frac{\partial z}{\partial x}\right)_{\perp}^{\top}\right]$ has full rank.
}

Proposition 4 implies that if there is a solution to the conditions of Propositions 1 and 3 with (17), then there also exists an (asymptotically) stabilizing control law (16). In addition, if $F_{2}=F_{20}=F_{21}$ then (16) is reduced to (6) and (17) becomes (7) (or (10)). Proposition 4 can be easily extended to work with Prop. 2 (instead of 1). In this case, (17) is reduced to

$$
\begin{gathered}
{\left[\begin{array}{ccc}
L_{i_{1}}^{\top} e_{1} & \ldots & L_{i_{m}}^{\top} e_{m}
\end{array}\right]+\left[\begin{array}{lll}
L_{i_{1}}^{\top} e_{1} & \ldots & L_{i_{m}}^{\top} e_{m}
\end{array}\right]^{\top} \prec 0,} \\
F_{2 i}=\left[\begin{array}{ll}
-g^{\top}(x) \Gamma(x) F_{1}^{\top}(x), & L_{i}(x)
\end{array}\right]\left[\begin{array}{l}
g_{\perp} \\
g^{\top}
\end{array}\right]^{-\top} \Gamma^{-\top}(x) .
\end{gathered}
$$

In the same way as in ( $\mathrm{Hu}$ and Lin, 2001; Valmorbida et al., 2013; Ichihara, 2013) for multiple input systems, we can adopt the independent input saturation given by $u_{\text {sat-i }}=\operatorname{sat}\left(u_{x}, \bar{u}, \underline{u}\right)$ and $u_{x}=u_{b}+\left(g^{\top} g\right) u_{\delta}$, where $\bar{u}$ and $\underline{u}$ are maximum and minimum values of $u_{b}$ in $\mathscr{U}_{b}$. Figure 1 illustrates the situation for $m=2$, $g^{\top} g=I_{2}, u_{x}, u_{b}, u_{\text {sat-i }}$ and sets $\mathscr{U}_{b}, \mathscr{U}_{s}$.

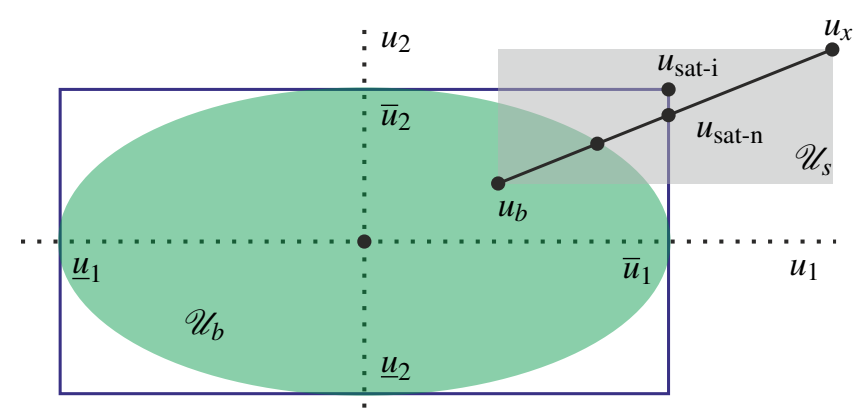

Figura 1. Relations of $u$ constrained and saturated.

Here, we also observed that in order to have AS, independent input saturation $\left(u_{\text {sat- }-1}\right)$ is not the only solution. Therefore, to simplify (17), we select $\theta_{1}=\theta_{2}=\cdots=\theta_{m}$ and a new saturation function given by

$$
\begin{aligned}
& u_{\text {sat-n }}= u_{b}+\left(g^{\top} g\right)^{-1} u_{\delta} \min \left(\rho_{1}, \ldots, \rho_{m}\right), \\
& \rho_{k}=\left\{\begin{array}{lll}
\frac{e_{k}^{\top}\left(\bar{u}-u_{b}\right)}{e_{k^{\top}}^{\top}\left(g^{\top} g\right) u_{\delta}}, & \text { if } e_{k}^{\top} u_{x}>e_{k}^{\top} \bar{u}, \quad e_{k}^{\top}\left(g^{\top} g\right) u_{\delta} \neq 0, \\
\frac{e_{k}^{k}\left(\underline{u}-u_{b}\right)}{e_{k}^{\top}\left(g^{\top} g\right) u_{\delta}}, & \text { if } e_{k}^{\top} u_{x}<e_{k}^{\top} \underline{u}, \quad e_{k}^{\top}\left(g^{\top} g\right) u_{\delta} \neq 0, \\
1, & \text { otherwise, }
\end{array}\right.
\end{aligned}
$$

which is also shown in Figure 1. Selection of $u_{\text {sat-n }}$ reduce $2^{m-1}$ inequalities and polynomial matrices $\bar{S}_{\star}$ in (17).

\subsection{Optimization Objectives in SDP}

Proposition 1-3 only guarantee a solution for $P$ and $F_{2}(x)$ without any performance or optimization goal in the SDP. In addition, we may set the following simple objectives:

\section{Optimization 1 (Volume maximization of $\mathscr{X}_{P}$ )}

$$
\begin{array}{cc}
\text { minimize } & \operatorname{trace}(Y) \\
\text { subject to } & {\left[\begin{array}{cc}
P & I_{n_{z}} \\
I_{n_{z}} & Y
\end{array}\right] \succeq 0 .}
\end{array}
$$

Proof 5 The volume of $\mathscr{X}_{P}$ is proportional to $\sqrt{\operatorname{det}(P)}$, see (Boyd et al., 1994, pp. 48-49). In addition, from KL-divergence between 
two multivariate normal distributions, we obtain the relation

$$
\operatorname{trace}\left(I_{n_{z}}-A^{-1}\right) \preceq \log (\operatorname{det}(A)) \preceq \operatorname{trace}\left(A-I_{n_{z}}\right)
$$

for any real matrix $A \succ 0$. Therefore, maximizing the volume of $\mathscr{X}_{P}$ with $P \succ 0$ is equivalent to maximize $\log (\operatorname{det}(P))$. Using (21) we enlarge the minimum bound of $\log (\operatorname{det}(P))$ by minimization of trace $\left(P^{-1}\right)$ which is equivalent to Opt. 1 with Schur complement in $(20)$.

This minimization is also used empirically in (Ichihara, 2013). Optimization 1 maximizes the volume of $\mathscr{X}_{P}$ by maximizing the minimum bound of $P$ given by $Y^{-1}$. Note that searching for the biggest $\mathscr{X}_{P}$ does not demand the explicit selection of $x_{0}$ (right hand side of (8)).

\section{Optimization 2 (Volume minimization of $\mathscr{U}_{b}$ )}

$$
\begin{aligned}
\text { minimize } & \text { trace }\left(S_{u}\right) \\
\text { subject to } & S_{u}=\text { constant. }
\end{aligned}
$$

Proof 6 Along the same lines of Optimization 1, except that we consider the upper bound of (21).

Without loss of generality, define $F_{2}(x)=\bar{F}_{2}(x) P N(x), 0=$ $\bar{F}_{2}(x) P\left(\frac{\partial z}{\partial x}\right)_{\perp}^{\top}(x)$, for some function $\bar{F}_{2} \in \mathbb{R}^{m \times n_{z}}$. Then, (14) be-
comes

$$
\left(g^{\top} g\right) S_{u}\left(g^{\top} g\right)-\left(\bar{F}_{2}-\Lambda_{2}\right) P\left(\bar{F}_{2}-\Lambda_{2}\right)^{\top} \succeq 0,
$$

for all $x \in \mathscr{X}_{h}$. This shows that minimization of $S_{u}$ (upper bound of $u$ ) is equivalent to minimize $\bar{F}_{2}-\Lambda_{2}$ and an upper bound of $P$. As a consequence, it is required to have at least one minimum bound on $P$ (right hand side of (8) or Opt. 1).

\section{Simulations}

It is well-known that the SOS property is a sufficient condition for checking the non-negativity of a polynomial function (Parrilo, 2000). For this reason, we may search for positive semidefinite matrices that are matrix SOS polynomials in Propositions 1-4. To guarantee strict inequalities in the SDP solver, we add $10^{-3} I_{n_{z}}$ in $P \succeq 0,10^{-3} I_{n_{x}-m}$ in (10), and $10^{-3} I_{n}$ in (7) and (17). The algorithm is processed in Matlab by use of SOSTOOLS and SDPT3, see (Papachristodoulou et al., 2016). For details on the transformation from SOS to SDP see (Parrilo, 2000).

In the following examples we search for asymptotically stabilizing controllers wrt. two systems using the results of Proposition 1-4. Values presented in this paper have been rounded to three decimals for better visibility.

\subsection{Nonlinear Second Order System}

We shall test Proposition 1-3 for synthesizing an asymptotically stabilizing constrained controller in the system

$$
\left[\begin{array}{c}
\dot{x}_{1} \\
\dot{x}_{2}
\end{array}\right]=\left[\begin{array}{c}
x_{1}^{2}+x_{2} \\
x_{1}
\end{array}\right]+\left[\begin{array}{l}
0 \\
1
\end{array}\right] u
$$

First, we pick $z(x)=\left[x_{1}, x_{1}^{2}+x_{2}\right]^{\top}, g_{\perp}=[1,0], \Gamma=I_{2}, \Lambda_{1}=[0,1]$ and $\Lambda_{2}=[1,0]$. Thus, $\frac{\partial z}{\partial x}$ is unimodular and C1-C2 are satisfied. Then we select $S_{h}=\operatorname{diag}(9,9), S_{1}(x) \in \mathbb{R}^{2 \times 2}$ with polynomials of degree 2 as elements and test Proposition 1 with Optimization 1 (maximizing $\mathscr{X}_{P}$ ), obtaining $\mathscr{X}_{P}=\left\{x \in \mathbb{R}^{2} \mid \gamma(x) \leq 1\right\}$, with $\gamma(x)=9 x_{1}^{4}-0.001 x_{1}^{3}+18 x_{1}^{2} x_{2}+9.0 x_{1}^{2}-0.001 x_{1} x_{2}+9 x_{2}^{2}$.

Next, for illustration we select (a minimum bound on $P$ ) $x_{0}^{\top}=[0,2] \in \mathscr{X}_{P}$ (previously found) and solve (for a new $P$ and $F_{2}$ ) the conditions of Prop. 1 and 3 with Opt. 2 (minimization of $S_{u}$ ) for $S_{3}(x) \in \mathbb{R}$ a polynomial of degree 6 , resulting in $S_{u}=100.134$.

Finally, we evaluate Prop. 1 and 3 with Opt. 1 selecting, for instance, $\mathscr{U}_{b}=\left\{u \in \mathbb{R} \mid S_{u}=11^{2} \geq\|u\|^{2}\right\}$. The results can be seen in Figure 2, which shows sets $\mathscr{X}_{P} \subset \mathscr{X}_{h}, \mathscr{X}_{P} \subset \mathscr{U}_{b}$, and the phase portrait in $x_{1}-x_{2}$ plane of the closed-loop for 10 extreme initial positions $x_{0}$ represented by symbol “ $*$ ”. Here all trajectories converge to the origin as expected. In addition, Figure 3 illustrates 5 seconds of respective control actions (calculated with (6)), which are all constrained in $\mathscr{U}_{b}$. As mentioned in Section 4, we can also use Prop. 2-3. Table 1 shows a comparison between both Propositions for $x_{0}^{\top}=[0,2] \in \mathscr{X}_{P}, S_{u}=11^{2}$. We conclude that Prop. 2 yields better optimization results.

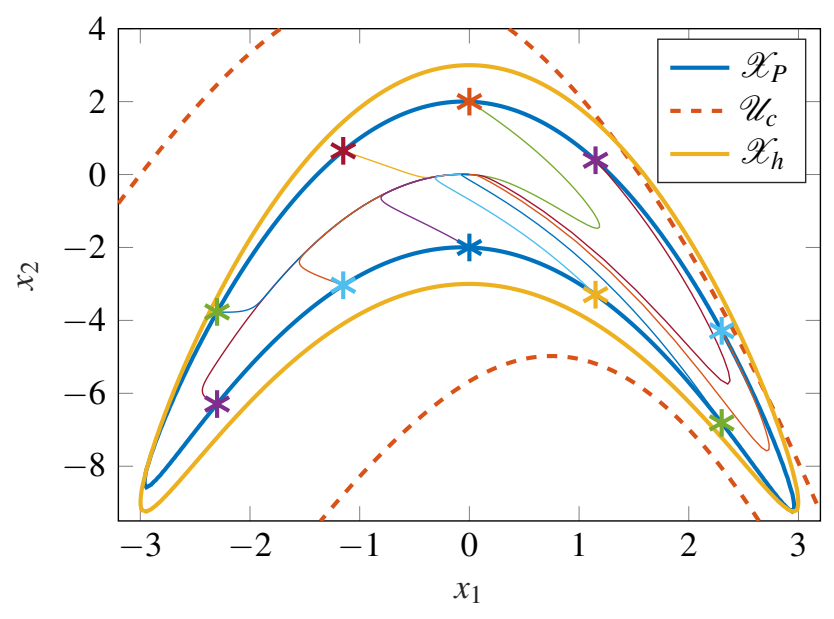

Figura 2. Sets $\mathscr{X}_{P}, \mathscr{X}_{h}, \mathscr{U}_{b}$, and phase portrait for 10 extreme initial positions.

Tabla 1. Comparison of Prop. 1, 2 in Sec. 5.1.

\begin{tabular}{lcc} 
& Prop. 1, 3 & Prop. 2, 3 \\
\hline Opt. 1: $\operatorname{det}(P)$ & 35.198 & 41.064 \\
Opt. 2: $S_{u}$ & 100.134 & 87.345 \\
\hline
\end{tabular}

\subsection{Third Order Multiple Input System with AS}

Now, we consider a third order system given by

$$
\left[\begin{array}{ccc}
1 & x_{1} & 0 \\
-x_{1} & 1 & 0 \\
0 & 0 & 1
\end{array}\right]\left[\begin{array}{c}
\dot{x}_{1} \\
\dot{x}_{2} \\
\dot{x}_{3}
\end{array}\right]=\left[\begin{array}{c}
x_{2}-2 x_{3}-2 x_{1} x_{2}-2 x_{2}^{2} \\
x_{1} x_{2}+u_{1} \\
x_{2} x_{3}+u_{2}
\end{array}\right]
$$




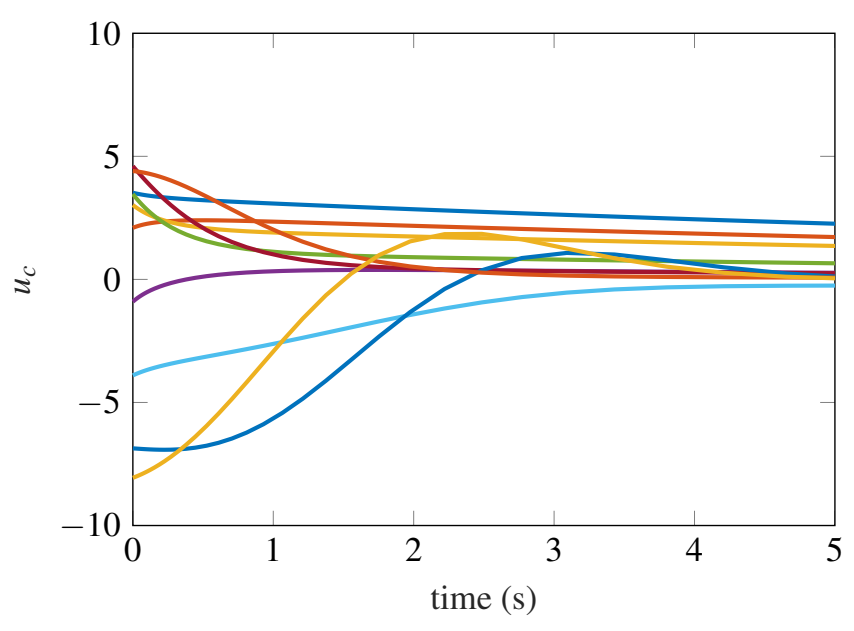

Figura 3. Response of control signal $u_{b}\left(u_{b}\right.$ stays in $\left.\mathscr{U}_{b}\right)$.

with AS using $u_{\text {sat-n }}$, i.e. $\theta_{1}=\theta_{2}$. In the controller synthesis according to $\mathrm{C} 1-\mathrm{C} 2$, we choose $g_{\perp}=[1,0,0]$,

$\Lambda_{1}^{\top}=\left[\begin{array}{c}0 \\ 1 \\ -2\end{array}\right], \quad \Lambda_{2}(x)=\left[\begin{array}{ccc}x_{2} & 0 & 0 \\ 0 & x_{3} & 0\end{array}\right], \quad \Gamma=\left[\begin{array}{ccc}1 & x_{1} & 0 \\ -x_{1} & 1 & 0 \\ 0 & 0 & 1\end{array}\right]$,

and $z(x)=\left[x_{1}, x_{2}, x_{2}^{2}+x_{1} x_{2}+x_{3}\right]^{\top}$ (unimodular $\frac{\partial z}{\partial x}$ ). This example is computationally more challenging. Therefore, we select $S_{h}=\operatorname{diag}(100,100,100)$ and use Prop. 2 as fast indicator (that Prop. 1 will work), which is met successfully. Then, we take $S_{1} \in \mathbb{R}^{3 \times 3}, F_{20}(x), F_{21}(x) \in \mathbb{R}^{2 \times 3}$ and $S_{3}(x) \in \mathbb{R}^{2 \times 2}$ with polynomials of degree 2 and 4 , respectively, and apply Propositions 1 , 3,4 with Opt. 1 for the user defined ${ }^{7}$ choice $S_{u}=\operatorname{diag}\left(10^{2}, 8^{2}\right)$.

For avoiding excessively large $u_{x}$, we constrain each of the constant elements of $F_{21}$ represented by $f_{i j}$ with $\left|f_{i j}\right|<10$. The results are illustrated in Figures 4 and 5 . Figure 4 shows the states ( $x_{1}$ scaled for clarity) in closed-loop under initial condition $[0,-0.65,0]^{\top}=x_{0} \in \mathscr{X}_{P} \subset \mathscr{X}_{h}$. It is clearly seen that all states will converge to the origin. Figure 5 illustrates the first second of $u_{\text {sat-n }}$. Note that $u_{2}$ is saturated, obviously, without compromising stability. Furthermore, using Prop. 2 in this system gives worse optimization results, which shows that the selection of the best Proposition (1 or 2) is system dependent.

\section{Conclusion}

In this paper we provide an algebraic solution for IDA-PBC that is able to resolve the problem of actuator saturation. To this end, we restrict the design to a class of polynomial systems that yield conditions which are solvable with SOS and SDP. The presented algorithm requires the following steps:

S1 Select $\Lambda_{1}, \Lambda_{2}, z, g_{\perp}$ and $h$.

S2 Define $S_{u}$ and calculate $u_{b}$ with Propositions 1 (or 2), 3 and Opt. 1 to maximize the volume of $\mathscr{X}_{P}$. The minimum $S_{u}$ can also be calculated with Opt. 2 .

\footnotetext{
${ }^{7}$ The minimum $S_{u}$ can be found similarly as in Example 5.1.
}

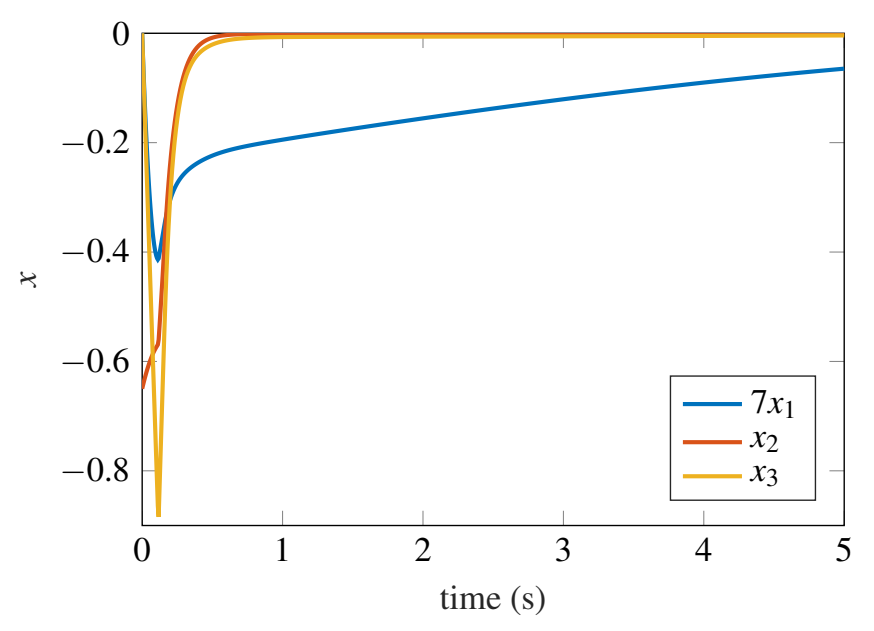

Figura 4. States with $x_{0}=[0,-0.65,0]^{\top}$ for the third order system in closed loop.

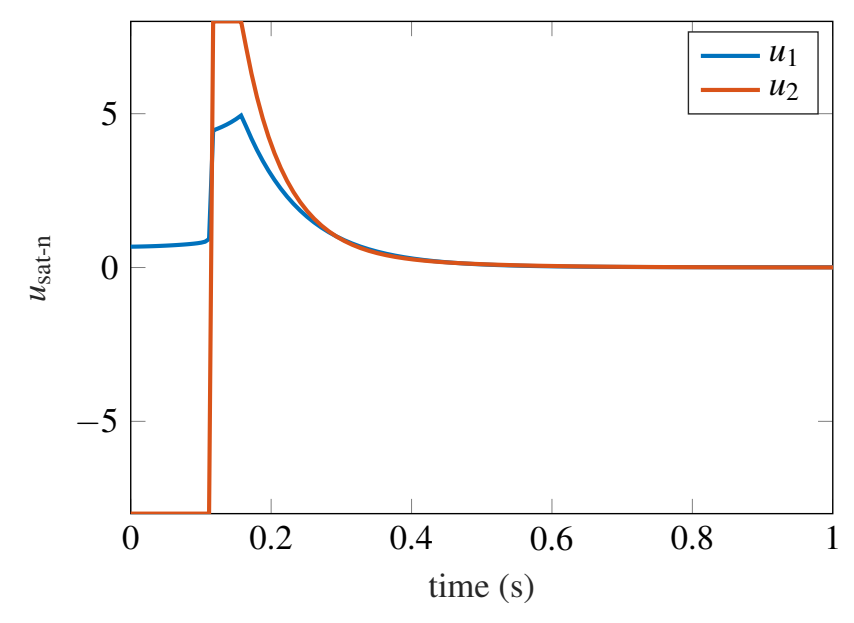

Figura 5. Saturated control action $u_{\text {sat-n }}$.

\section{S3 Compute $u_{\delta}$ with Prop. 4 and $P, F_{2}$ found in S2.}

S4 Implement the saturation functions $u_{\text {sat-i }}$ or $u_{\text {sat-n }}$.

Additionally, we enjoy features as: no need to solve a PDE, dissipation in design, and one step IDA-PBC. Simulations of two polynomial example systems validate our approach.

\section{ACKNOWLEDGMENT}

The first author would like to acknowledge the financial support obtained from: (1) Deutscher Akademischer Austauschdienst, Germany, and (2) Programa Nacional de Becas y Crédito Educativo, Peru. The second author would like to acknowledge the financial support from European Union Horizon 2020 research and innovation program, Marie Skłodowska-Curie grant agreement No. 734832 .

\section{REFERENCES}

Astolfi, A., Chhabra, D., and Ortega, R. (2002a). Asymptotic Stabilization of Some Equilibria of an Underactuated Underwater Vehicle. Systems \& Control Letters, 45:193-206. 
Astolfi, A. and Ortega, R. (2001). Energy-Based Stabilization of Angular Velocity of Rigid Body in Failure Configuration. Journal of Guidance, Control, and Dynamics, 25(1):184-187.

Astolfi, A., Ortega, R., and Sepulchre, R. (2002b). Stabilization and Disturbance Attenuation of Nonlinear Systems Using Dissipativity Theory. European Journal of Control, 8(5):408-431.

Åström, K. J., Aracil, J., and Gordillo, F. (2008). A family of smooth controllers for swinging up a pendulum. Automatica, 44(7):1841-1848.

Batlle, C., Dòria-Cerezo, A., Espinosa-Pérez, G., and Ortega, R. (2007). Simultaneous interconnection and damping assignment passivity-based control: Two practical examples. In Lagrangian and Hamiltonian Methods for Nonlinear Control 2006, pages 157-169. Springer.

Batlle, C., Doria-Cerezo, A., and Ortega, R. (2004). Power flow control of a doubly-fed induction machine coupled to a flywheel. In International Conference on Control Applications, volume 2, pages 1645-1650.

Borja, P., Cisneros, R., and Ortega, R. (2016). A constructive procedure for energy shaping of port-Hamiltonian systems. Automatica, 72(1):230-234.

Boyd, S., El Ghaoui, L., Feron, E., and Balakrishnan, V. (1994). Linear Matrix Inequalities in System and Control Theory, volume 15. SIAM studies in Applied Mathematics.

Castaños, F. and Gromov, D. (2016). Passivity-Based Control of Implicit Port-Hamiltonian Systems with Holonomic Constraints. Systems \& Control Letters, 94(1):11-18.

Cieza, O. B. and Reger, J. (2018). IDA-PBC for Polynomial Systems : An SOS-based Approach. In IFAC Conference on Modelling, Identification and Control of Nonlinear Systems (MICNON), pages 366-371.

Delgado, S. and Kotyczka, P. (2014). Overcoming the Dissipation Condition in Passivity-based Control for a class of mechanical systems. In IFAC World Congress, pages 11189-11194.

Donaire, A., Mehra, R., Ortega, R., Satpute, S., Romero, J. G., Kazi, F., and Singh, N. M. (2016a). Shaping the Energy of Mechanical Systems Without Solving Partial Differential Equations. IEEE Transactions on Automatic Control, 61(4):1051-1056.

Donaire, A., Ortega, R., and Romero, J. G. (2016b). Simultaneous Interconnection and Damping Assignment Passivity-based Control of Mechanical Systems Using Generalized Forces. Systems \& Control Letters, 94(1):118-126.

Escobar, G., Ortega, R., and Sira-Ramírez, H. (1999). Output Feedback Global Stabilization of a Nonlinear Benchmark System Using a Saturated Passivity Based Controller. IEEE Transactions on Control Systems Technology, 7(2):289-293.

Fujimoto, K., Sakurama, K., and Sugie, T. (2001). Trajectory tracking control of port-controlled Hamiltonian systems and its application to a magnetic levitation system. In Conference on Decision and Control, pages 3388-3393.
Fujimoto, K. and Sugie, T. (2001). Canonical transformation and stabilization of generalized Hamiltonian systems. Systems \& Control Letters, 42(9):217-227.

Gómez-Estern, F. and Van der Schaft, A. (2004). Physical Damping in IDA-PBC Controlled Underactuated Mechanical Systems. European Journal of Control, 10(5):451-468.

Hu, T. and Lin, Z. (2001). Control Systems with Actuator Saturation: Analysis and Design. Birkhäuser.

Ichihara, H. (2009). Optimal Control for Polynomial Systems Using Matrix Sum of Squares Relaxations. IEEE Transactions on Automatic Control, 54(5):1048-1053.

Ichihara, H. (2013). A Convex Approach to State Feedback Synthesis for Polynomial Nonlinear Systems with Input Saturation. SICE Journal of Control, Measurement, and System Integration, 6(3):186-193.

Jennawasin, T., Kawanishi, M., Narikiyo, T., and Lin, C.-L. (2012). An Improved Stabilizing Condition for Polynomial Systems with Bounded Actuators: An SOS-Based Approach. In IEEE International Symposium on Intelligent Control (ISIC), pages 258-263. IEEE.

Jennawasin, T., Narikiyo, T., and Kawanishi, M. (2010). An improved SOS-based stabilization condition for uncertain polynomial systems. In SICE Annual Conference 2010, pages 30303034.

Li, H., Wang, X., and Tian, T. (2010). The performance research of induction motor systems controlled by the IDA-PBC method and its speed sensorless implementation. In International Conference on Electrical Machines and Systems (ICEMS), pages 680-683.

Li, J., Liu, Y., Li, C., and Chu, B. (2013). Passivity-based nonlinear excitation control of power systems with structure matrix reassignment. Information, 4(3):342-350.

Macchelli, A. (2002). Port Hamiltonian systems: A unified approach for modeling and control finite and infinite dimensional physical systems. Ph.d. dissertation, University of Bologna.

Macchelli, A. (2014). Passivity-Based Control of Implicit PortHamiltonian Systems. SIAM Journal on Control and Optimization, 52(4):2422-2448.

Macchelli, A., Melchiorri, C., Secchi, C., and Fantuzzi, C. (2003). A variable structure approach to energy shaping. In European Control Conference (ECC), pages 1309-1314.

Nunna, K., Sassano, M., and Astolfi, A. (2015). Constructive Interconnection and Damping Assignment for Port-Controlled Hamiltonian Systems. IEEE Transaction on Automatic Control, 60(9):2350-2361.

Ortega, R. and García-Canseco, E. (2004). Interconnection and Damping Assignment Passivity-Based Control: A Survey. European Journal of Control, 10(5):432-450. 
Papachristodoulou, A., Anderson, J., Valmorbida, G., Prajna, S., Seiler, P., and Parrilo, P. A. (2016). SOSTOOLS: Sum of squares optimization toolbox for MATLAB. User's guide.

Parrilo, P. A. (2000). Structured Semidefinite Programs and Semialgebraic Geometry Methods in Robustness and Optimization. $\mathrm{PhD}$ thesis, California Institute of Technology.

Petrović, V., Ortega, R., and Stanković, A. M. (2001). Interconnection and damping assignment approach to control of PM synchronous motors. IEEE Transactions on Control Systems Technology, 9(6):811-820.

Renton, C., Teo, Y. R., Donaire, A., and Perez, T. (2012). Active control of car suspension systems using IDA-PBC. In Australian Control Conference, pages 361-366.

Romero, J. G., Donaire, A., Ortega, R., and Borja, P. (2017). Global Stabilisation of Underactuated Mechanical Systems via PID Passivity-Based Control. In IFAC World Congress, pages 95779582.

Sepulchre, R., Janković, M., and Kokotović, P. V. (1997). Constructive nonlinear control. Springer.

Sprangers, O., Lopes, G. A. D., and Babuska, R. (2015). Reinforcement learning for port-Hamiltonian systems. IEEE Transactions on Cybernetics, 45(5):1017-1027.

Sun, W., Lin, Z., and Wang, Y. (2009). Global Asymptotic and Finite-gain $\mathscr{L}_{2}$ Stabilization of Port-Controlled Hamiltonian Systems Subject to Actuator Saturation. In American Control Conference (ACC), pages 1894-1898.

Valmorbida, G., Tarbouriech, S., and Garcia, G. (2013). Design of polynomial control laws for polynomial systems subject to actuator saturation. IEEE Transactions on Automatic Control, 58(7):1758-1770.

Viola, G., Ortega, R., Banavar, R., Acosta, J. A., and Astolfi, A. (2007). Total Energy Shaping Control of Mechanical Systems: Simplifying the Matching Equations Via Coordinate Changes. IEEE Transactions on Automatic Control, 52(6):1093-1099.

Wei, A. and Yuzhen, W. (2010). Stabilization and $\mathscr{H}_{\infty}$ control of nonlinear port-controlled Hamiltonian systems subject to actuator saturation. Automatica, 46(12):2008-2013.

Wibowo, B. S., Trilaksono, B. R., and Syaichu-Rohman, A. (2014). $H_{\infty}$ Control of Polynomial Fuzzy Systems: A Sum of Squares Approach. Journal of Engineering and Technological Sciences, 46(2):152-169.

Xue, L. and Zhiyong, G. (2017). Control of Underactuated Bridge Cranes: A Simplified IDA-PBC Approach. In 11th Asian Control Conference (ASCC), pages 717-722.

Yu, A. G.-R. and Wang, B. S.-M. (2013). Polynomial Fuzzy Control of an Inverted Pendulum System by Sum-of-Squares Approach. In IEEE International Symposium on Next-Generation Electronics (ISNE), pages 236-239. IEEE.
Zhu, Y., Zhao, D., Yang, X., and Zhang, Q. (2018). Policy Iteration for $\mathscr{H}_{\infty}$ Optimal Control of Polynomial Nonlinear Systems via Sum of Squares Programming. IEEE Transactions on Cybernetics, 48(2):500-509.

\section{BIOGRAPHIES}

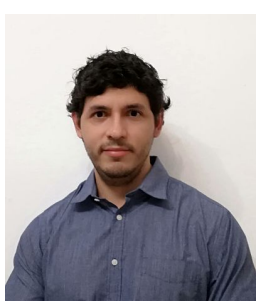

Oscar B. Cieza A. received the B.Sc. degree in Electronics Engineering in 2011 from the Pontificia Universidad Católica del Perú, Lima, and in 2014 the M.Sc. double degree in Mechatronics Engineering from the Technische Universität Ilmenau, Germany, and the Pontificia Universidad Católica del Perú. He is a doctoral student with the Control Engineering Group at TU Ilmenau since October 2015. His research interests include Interconnection and damping assignment passivitybased control (IDA-PBC) as well as robust control of underactuated mechanical systems.

Dr. Johann Reger received his diploma degree (Dipl.-Ing.) in Mechanical Engineering in 1999 and his doctorate (Dr.-Ing.) in Control Engineering in 2004, both from the University of Erlangen-Nuremberg in Germany. He has held several postdoc positions, among others, with the Mechatronics Department at CINVESTAV-IPN in Mexico-City, the EECS Control Laboratory at the University of Michigan in Ann Arbor, and the Control Systems Group at TU Berlin. Since 2008 he is a full professor and head of the Control Engineering Group at the Computer Science and Automation Faculty, TU Ilmenau, in Germany. There he also serves as vice-dean and director of the Institute for Automation and Systems Engineering. His current research foci are on adaptive and robust control, variable structure and sliding mode control, state and parameter estimation. Application areas include robotics, mechatronics, automotive, and water systems. 